\title{
Ultrasound, X-ray, computed tomography and clinical tests for diagnosis of abdominal purpura in children: A retrospective study
}

\author{
YURONG GE ${ }^{1^{*}}$ and SHUCHUN LIU ${ }^{2 *}$ \\ ${ }^{1}$ Department of Ultrasonic Medicine, Guiyang Maternal and Child Health Hospital, Guiyang, Guizhou 550003; \\ ${ }^{2}$ Department of Anesthesiology, Guizhou Provincial People's Hospital, Guiyang, Guizhou 550002, P.R. China
}

Received May 15, 2019; Accepted February 28, 2020

DOI: $10.3892 /$ etm.2020.8643

\begin{abstract}
Imaging modalities are not included in The European League Against Rheumatism, The Pediatric Rheumatology International Trials Organization and The Pediatric Rheumatology European Society 2010 criteria for the evaluation of abdominal purpura. The objective of the present study was to compare diagnostic parameters of ultrasound, X-ray and computed tomography (CT) for diagnosis of abdominal purpura considering the American College of Rheumatology (ACR) criteria as 'reference standard' in children with acute abdominal pain. Medical records of 215 children with acute abdominal pain were reviewed. Data regarding demographics and clinical characteristics, laboratory tests, X-ray, ultrasound findings, and computed tomographic images were collected and analyzed. Decision curve analysis was used for evaluation of the beneficial score for each diagnostic modality. Among diagnostic modalities, CT had the highest sensitivity (0.939); however, ultrasound findings had the highest accuracy (0.861) for diagnosis of abdominal purpura. Unlike X-ray and laboratory tests, ultrasound and CT were successful at detecting abdominal purpura when children had only colicky pain and were aged $<20$ years; however, occult blood stool test and granulocytes in the walls of small venules and arterioles (biopsy results) were negative. With respect to the ACR criteria, there were seven and three inconclusive results for ultrasound and CT,
\end{abstract}

Correspondence to: Dr Shuchun Liu, Department of Anesthesiology, Guizhou Provincial People's Hospital, 83 Zhongshan East Road, Nanming, Guiyang, Guizhou 550002, P.R. China

E-mail: sloanekingljw@yahoo.com

Dr Yurong Ge, Department of Ultrasonic Medicine, Guiyang Maternal and Child Health Hospital, 63 Ruijin Middle Road, Yunyan, Guiyang, Guizhou 550003, P.R. China

E-mail: 707970257@qq.com

*Contributed equally

Key words: American College of Rheumatology criteria, abdominal purpura, biopsy, computed tomography, occult blood stool test, ultrasound, X-ray respectively. Abdominal ultrasound is an easy, non-invasive and safe method for the detection of abdominal purpura in children.

\section{Introduction}

Abdominal purpura is inflammation of systematic small vessels of unknown etiology (1) and common in individuals aged $<20$ years (2). It is difficult to diagnose abdominal purpura if children only have abdominal pain (3) because abdominal purpura is an acute, complex, immune-mediated vasculitis characterized by abdominal pain (4), purpuric skin lesions (5), and bloody diarrhea (6) only without thrombocytopenia (7). Differences in the clinical characteristics and pathogenesis for abdominal purpura among Xining, Tibet, inner Mongolia, and other areas were previously reported (8).

According to The American College of Rheumatology (ACR) criteria, two out of four criteria (acute abdominal pain, age $\leq 20$ years or less, bowel angina, and granulocytes in the walls of small venules and arterioles in the biopsies) must be met for diagnosis of abdominal purpura (6). These criteria have different sensitivities and specificities. Moreover, biopsies are often difficult to obtain and some patients can have atypical presentations. In addition, the age criteria are considered to be redundant (9). Lastly, imaging modalities are not used routinely in the evaluation of abdominal purpura (10).

$\mathrm{X}$-rays may suggest the presence of fecal impaction, an obstruction and duodenal atresia (11). Computed tomography (CT) scans of the abdomen and pelvis can provide a high yield of information (12). Ultrasound scans avoid exposure to radiation (11). There is a risk of overtreatment if children with abdominal pain are treated with steroids because steroids can cause a rapid reduction in abdominal pain but do not prevent recurrence (13). Steroids can also cause weight gain issues (4). Therefore, there is a need for non-invasive and accurate diagnostic modality for abdominal purpura.

In clinical practice, it is known that abdominal ultrasound is the best approach for the correct diagnosis of abdominal complications. However, in some cases, CT can provide more detailed information. Therefore, each diagnostic technique has its specific role.

The objectives of the present study were to compare diagnostic parameters of ultrasound, X-ray and CT for diagnosis of abdominal purpura, and to find additional parameters to 
evaluate and administer early treatment, considering ACR criteria as the 'gold standard (reference index test)' in children with acute abdominal pain in China.

\section{Patients and methods}

Compliance with ethical standards. The protocol of the present study was approved by the Guiyang Maternal and Child Health Hospital review board. The present study was a retrospective analysis of prospectively collected data. An informed consent form was signed by the parents of all participating children regarding diagnosis, radiology, treatment and anesthesia (if required) during hospitalization. The present study adheres to the law of China, the Strengthening the Reporting of Observational Studies in Epidemiology statement and the Declaration of Helsinki (2008).

Inclusion criteria. FromJanuary 1, 2013 to February 13, 2019, data from 227 children [109 (51\%) boys and 106 (49\%) girls] in the age range from 3-16 years (mean: 11.25 \pm 2.11 years) were available at the outpatient setting of Guiyang Maternal and Child Health Hospital. The children experienced acute abdominal pain or abdominal tenderness, and exhibited typical skin purpura.

Exclusion criteria. Among the available children, complete records of 12 children were not available. Therefore, they were excluded from the present study. Medical records of 215 children were included in the present analysis. A flow chart of the analysis is presented in Fig. 1.

Data collection. Data regarding demographical parameters, laboratory test results, X-ray, ultrasound findings, and CT images were collected of enrolled children.

Image examination. Dilatation and motility of the intestinal segments, intramural hemorrhage, and the wall of the small bowel were investigated by image examinations. X-ray and CT scans were carried out by radiologists with a minimum of 3 years of experience. Ultrasound was performed by gastroenterologists with a minimum of 3 years of experience.

Abdominal X-ray. Supine anteroposterior and/or horizontal beam (upright, decubitus or cross-table lateral) views of abdominal X-rays (Brivo XR115; GE Healthcare) were performed at $60-75 \mathrm{kVp}, 100 \mathrm{~mA}$ and $200 \mathrm{kHz}$.

Abdominal ultrasound. Abdominal ultrasound was performed using the Philip EPEQ5 5-13 MHz linear probe.

Abdominal CT. Abdominal CT was performed using the Revolution Apex scanner (GE Healthcare).

Beneficial score analysis. Decision curve analysis was used for evaluation of the beneficial score and working area for each diagnostic modality as follows:

The working area is defined as the likelihood to detect abdominal purpura. Sensitivity is the ratio of the value of true positive abdominal purpura detected by imaging modality with those detected by ACR criteria. Accuracy is the ratio

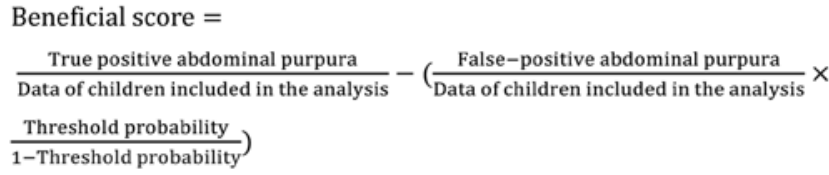

of the value of true negative abdominal purpura detected by imaging modality with those detected by ACR criteria.

Statistical analysis. SPSS Statistics version 25 (IBM Corp.) was used for statistical analysis. Categorical parameters are demonstrated as frequency (percentage) and continuous parameters are demonstrated as the mean \pm SD. Categorical parameters were analyzed using the $\chi^{2}$ test or the Fisher's exact test (14) and continuous parameters were analyzed using the Wilcoxon sum rank test or the Mann-Whitney U test. $\mathrm{P}<0.05$ was considered to indicate a statistically significant difference.

\section{Results}

Clinical features. In total, 99 (46\%) children had abdominal tenderness located in the periumbilical area. Nausea, vomiting and diarrhea were frequent among the children. Bloody diarrhea was present in 8 (4\%) children. Hematuria was observed in $18(8 \%)$ children.

Laboratory tests. All children had an erythrocyte sedimentation rate above the normal range, 33 (15\%) children had a positive occult blood stool test. All children were put on Deflazacort at $0.9 \mathrm{mg} / \mathrm{kg}$ orally once a day for 6-8 days. Other laboratory test results are presented in Table I.

$X$-ray measurements. Interior posterior views (Fig. 2A) and lateral views (Fig. 2B) of the abdomen were successful in the presentation of ileus and epididymitis (considering urinary bladder as a reference limit) but X-ray did not detect abdominal involvement where the occult blood stool test and biopsy results were negative (Fig. 2C).

Ultrasound measurements. Ultrasound examinations identified thickening of the bowel wall accompanied by reduced peristalsis and dilated bowel loops. An outer hypoechoic ring from the edematous walls of the intussuscipiens was identified around an echo-dense center of the intussuceptum under ultrasound examination of empty ileum $(n=85$; Fig. 3A). With confirmed ileocecal intussusception, subdiaphragmatic free gas was not found on the ultrasound scan of the duodenum $(n=42$; Fig. 3B). However, on the outer surface of the colon, thick loops with extensive linear hemorrhages and bowel perforation were identified ( $\mathrm{n}=22$; Fig. 3C).

CT measurements. Thickening of the wall and focal dilated small loop at the jejunum were detected in the abdominal CT scan (Fig. 4A). In addition, CT scans detected bowel perforation (Fig. 4B) and intussusception in abdomen (Fig. 4C).

Diagnostic parameters. CT had the highest sensitivity (0.939) but ultrasound findings had the highest accuracy (0.861) among diagnostic modalities for detection of abdominal purpura. The other diagnostic parameters of imaging modalities are presented in Table II. 
Table I. Laboratory test results.

\begin{tabular}{|c|c|c|c|}
\hline Test & Normal value & Test prediction & Test results \\
\hline Serum amylase, IU/1 & $23-85$ & Abnormal & $135 \pm 25$ \\
\hline Urine amylase, IU/1 & $20-400$ & Abnormal & $1,215 \pm 85$ \\
\hline Hemoglobin, g/dl & $12-15$ & Normal & $11.5 \pm 1.12$ \\
\hline Total leucocyte, count/ $\mu 1$ & $4,500-11,000$ & Abnormal & $13,125 \pm 245$ \\
\hline Platelet, count $/ \mu 1$ & $150,000-450,000$ & Normal & $353,561 \pm 7,245$ \\
\hline Erythrocytes sedimentation rate, $\mathrm{mm}$ per the first hour & $12-20$ & Abnormal & $28.12 \pm 3.45$ \\
\hline Serum immunoglobulin A, mg/dl & $40-230$ & Borderline & $210 \pm 35$ \\
\hline Protein urea, mg/mmol & $<100$ & & \\
\hline Positive & & & $116(54)$ \\
\hline Negative & & & $99(46)$ \\
\hline \multirow{2}{*}{$\begin{array}{l}\text { Granulocytes in the walls of small venules and arterioles } \\
\text { in the biopsies }\end{array}$} & $\mathrm{N} / \mathrm{A}$ & Positive & $65(30)$ \\
\hline & & Negative & $150(70)$ \\
\hline \multirow[t]{2}{*}{ Occult blood stool test } & $\mathrm{N} / \mathrm{A}$ & Positive & $33(15)$ \\
\hline & & Negative & $182(85)$ \\
\hline Treatment & N/A & N/A & $\begin{array}{l}\text { Deflazacort }(0.9 \mathrm{mg} / \mathrm{kg} \text { orally } \\
\text { once a day for } 6-8 \text { days })\end{array}$ \\
\hline Onset of symptoms, days & N/A & $\mathrm{N} / \mathrm{A}$ & $7 \pm 2$ \\
\hline
\end{tabular}

Constant data are presented as 'number (percentage)' and continuous data are presented as the mean \pm SD. n=215. N/A, not applicable.

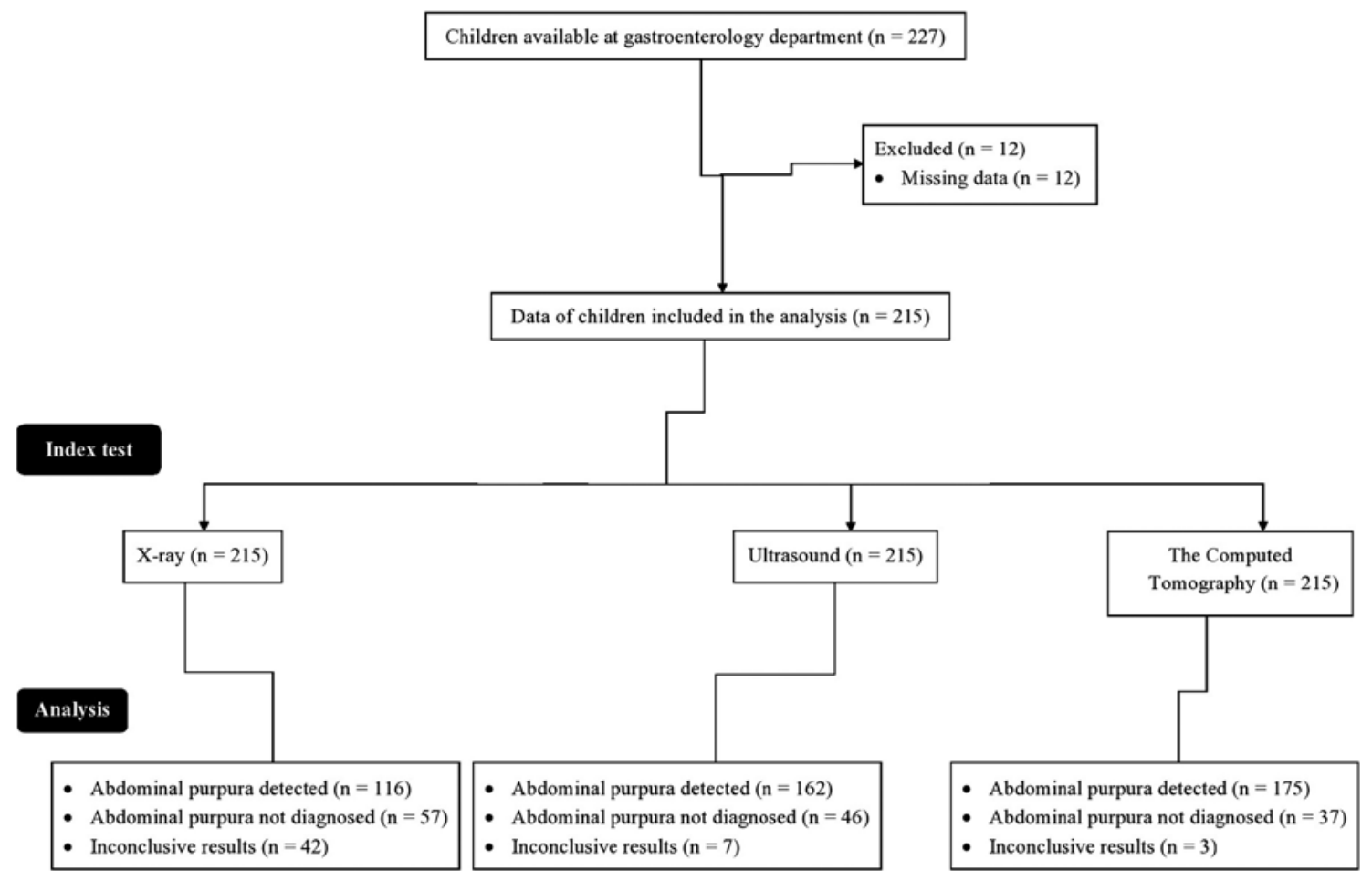

Figure 1. Flow chart of the analysis.

Beneficial score analysis. X-ray findings had 0-0.75 working area to detect abdominal purpura at one time and $>0.75$ thresholds (only colic pain with age $<20$ years, absent of the other laboratory tests) there was a risk of over-diagnosis and overtreatment. Ultrasound findings had 0-0.916 working area to detect abdominal purpura at one time and $>0.916$ thresholds there was a risk of over-diagnosis and over-treatment. CT had 0-0.922 working area to detect abdominal purpura at one time and $>0.922$ thresholds there was a risk of over-diagnosis and overtreatment. Ultrasound and CT were successful at 


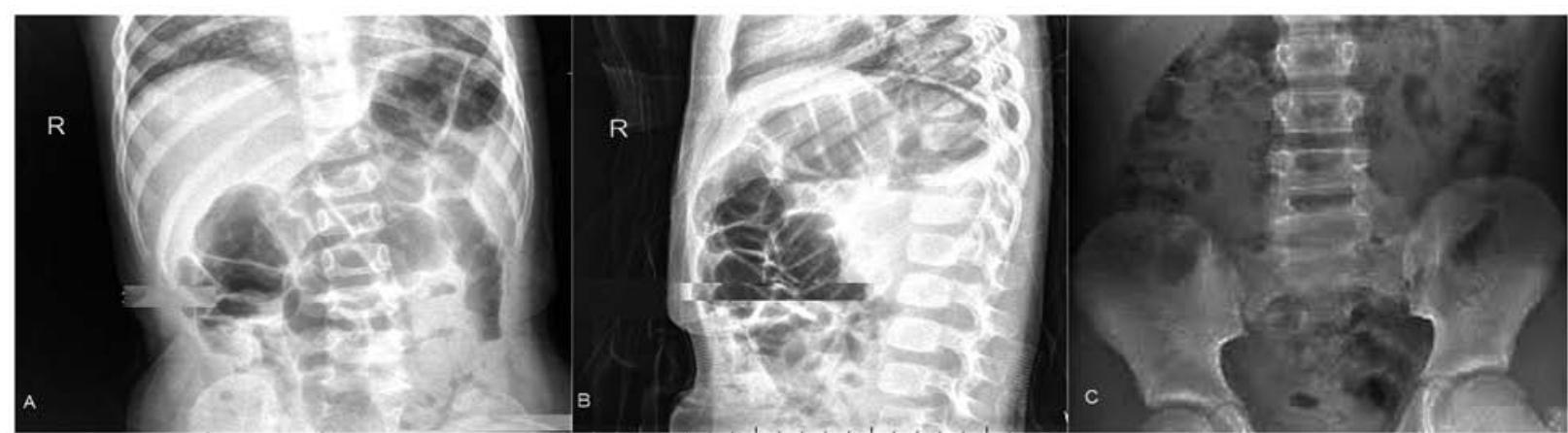

Figure 2. Abdominal X-ray measurements. (A) Interior posterior X-ray view of the abdomen of a child (age 8 years). Successful in the presentation of ileus and epididymitis. (B) Lateral X-ray view of the abdomen of a child (age 8 years). Successful in the presentation of ileus and epididymitis. (C) Interior posterior $\mathrm{X}$-ray view of the abdomen of a child (age 9 years). No detected abdominal involvement. The occult blood stool test and biopsy results were negative.

\section{A} B C

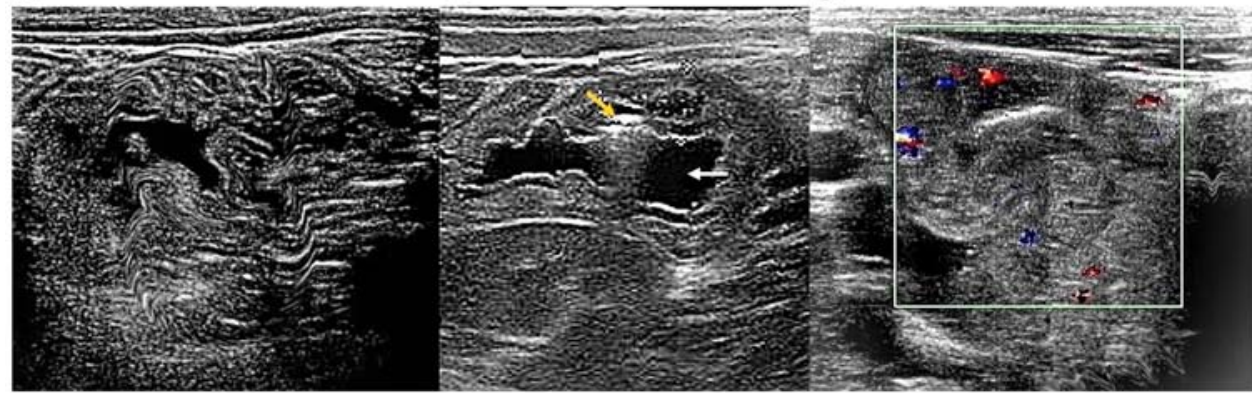

Figure 3. Abdominal ultrasound measurements. (A) Ultrasound scan of empty ileum of a child (age 8 years). Sagittal image of the abdomen. (B) Ultrasound scan of the duodenum of a child (age 8 years). Sagittal image of the abdomen. Hemorrhages (yellow arrow) and bowel perforation (white arrow) were detected. (C) Ultrasound scan of the colon of a child (age 8 years). Sagittal image of the abdomen. The hyperechoic submucosal layer (blue dots) and the hypoechoic muscularis layer (red dots) are shown.

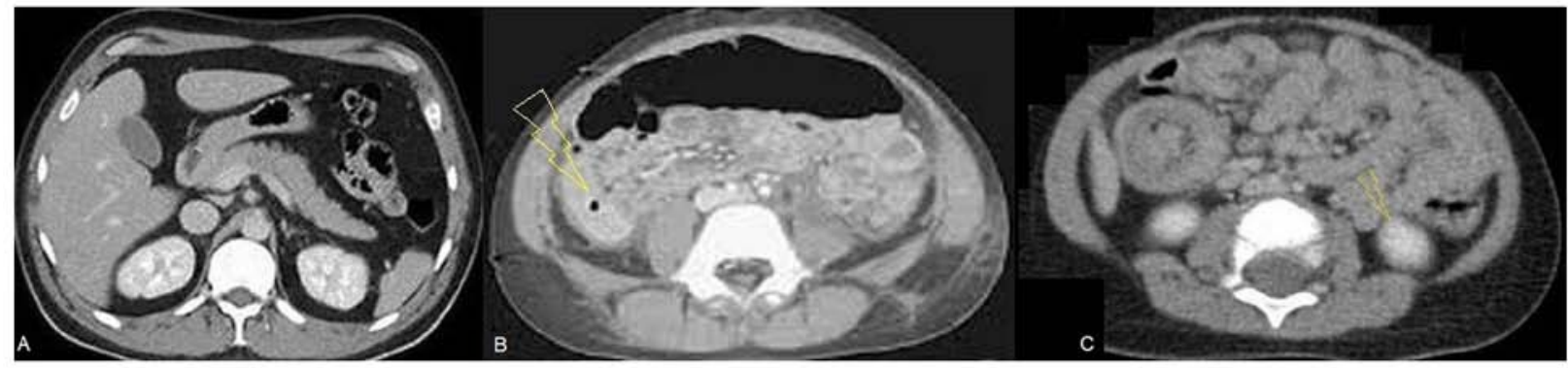

Figure 4. Abdominal CT measurements. (A) CT scan of the abdomen of a child (age 8 years). Thickening of the wall and focal dilated small loop at the jejunum. (B) CT scan of the abdomen of a child (age 7 years). The arrow indicates bowel perforation (yellow). (C) CT scan of the abdomen of a child (age 6 years). The arrow indicates intussusception in abdomen (yellow). CT, computed tomography.

detecting abdominal purpura when children had only colic pain and were aged $<20$ years; however; occult blood stool test and biopsy results were absent (Fig. 5).

\section{Discussion}

Laboratory tests and clinical features provide baseline information; however, do not rule out other etiologies of colicky pain, except occult blood stool test and biopsy results. However, biopsies are rarely preferred in the diagnosis of abdominal pain by physicians (15). Abdominal gastrointestinal symptoms are often observed prior to clinical symptoms (4). Ultrasound, CT and $\mathrm{X}$-ray provide direct insight into visualization and detection of complications and bowel involvement (for example, bowel perforation and intussusception) (13) but imaging criteria are not used in the diagnosis of purpura (9). Even The European League Against Rheumatism, The Pediatric Rheumatology International Trials Organization and The Pediatric Rheumatology European Society 2010 criteria (15), which are the current gold standard, do not include imaging criteria for the diagnosis of abdominal purpura (4). However, imaging modalities could play an important role in the diagnosis of abdominal purpura in children.

In the present study, ultrasound, CT and X-ray were successful at detecting abdominal purpura when children had colicky pain, aged $<16$ years, and had bowel angina. Ultrasound 
Table II. Diagnostic parameters.

Diagnostic modalities measurements

\begin{tabular}{|c|c|c|c|c|c|c|c|}
\hline \multirow[b]{3}{*}{ Parameters } & \multirow[b]{3}{*}{ ACR criteria ${ }^{a}$} & \multirow{2}{*}{\multicolumn{2}{|c|}{ X-ray }} & & & & \\
\hline & & & & \multicolumn{2}{|c|}{ Ultrasound } & \multicolumn{2}{|c|}{ Computed tomography } \\
\hline & & Result & $\mathrm{P}$-value & Result & P-value ${ }^{b}$ & Result & P-value ${ }^{b}$ \\
\hline True positive & $179(83)$ & $101(47)$ & $<0.0001$ & $154(72)$ & 0.006 & $168(78)$ & $0.223^{\mathrm{c}}$ \\
\hline True negative & $36(17)$ & $24(11)$ & $0.126^{\mathrm{c}}$ & $31(14)$ & $0.595^{\mathrm{c}}$ & $25(12)$ & $0.167^{\mathrm{c}}$ \\
\hline False positive & $0 \quad(0)$ & $35(16)$ & $<0.0001$ & 8 (4) & 0.013 & 7 (3) & 0.022 \\
\hline False negative & 0 & $22(10)$ & $<0.0001$ & $15(7)$ & 0.0002 & $12(6)$ & 0.001 \\
\hline Inconclusive results & $0 \quad(0)$ & $33(16)$ & $<0.0001$ & 7 (3) & 0.022 & $3(1)$ & $0.247^{\mathrm{c}}$ \\
\hline Sensitivity & 1 & 0.564 & $<0.0001$ & 0.86 & $<0.0001$ & 0.939 & $<0.0001$ \\
\hline Accuracy & 1 & 0.667 & $<0.0001$ & 0.861 & $<0.0001$ & 0.694 & $<0.0001$ \\
\hline
\end{tabular}

Constant data are presented as 'number (percentage)' and continuous data are presented as the mean. The $\chi^{2}$ independence test for constant data and the Wilcoxon sum rank test for continuous data were used for statistical analysis. $\mathrm{P}<0.05$ was considered to indicate a statistically significant difference. ${ }^{a} \mathrm{ACR}$ criteria: Two among four criteria (acute abdominal pain, aged $\leq 20$, bowel angina, and granulocytes in the walls of small venules and arterioles in the biopsies) are required for diagnosis. ${ }^{b}$ With respect to ACR criteria. ${ }^{c}$ Non-significant difference with respect to ACR criteria. ACR, American College of Rheumatology.

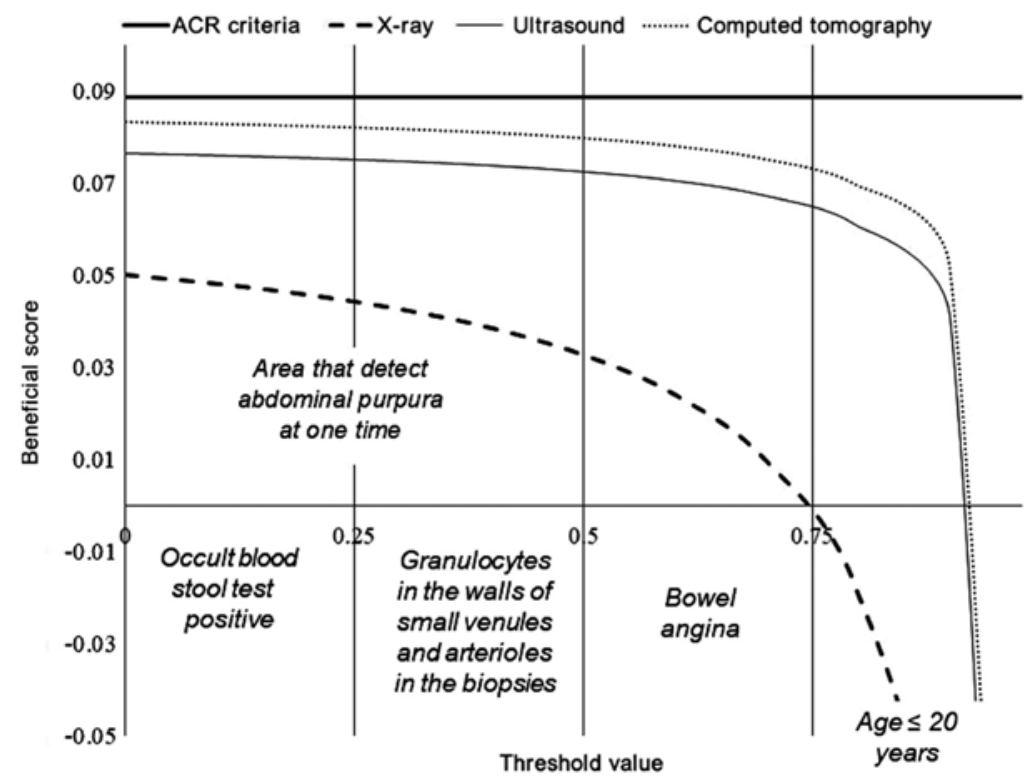

Figure 5. Decision curve analysis for the diagnosis of abdominal purpura. ACR criteria: Two among four criteria (acute abdominal pain, aged $\leq 20$ years, bowel angina, and granulocytes in the walls of small venules and arterioles in the biopsies) are required for diagnosis. ACR, American College of Rheumatology.

and CT had similar working areas but ultrasound had high accuracy among all imaging modalities. X-ray was not effective in cases where occult blood stool tests and biopsy results were negative. The results of the present study were consistent with a previous retrospective study (13) and a previous case report (3). The As Low As Reasonably Possible principle is required to maintain in the $\mathrm{CT}$ of children to avoid the risk of cancer (12), which is difficult to manage in children (16). Ultrasound has the ability to assess hematomas, mural thickness, small bowel intussusceptions and peritoneal fluid (3). Ultrasound provides an easy, non-invasive and safe method for the detection of abdominal purpura in children.

With respect to the ACR criteria, there were seven and three inconclusive results for ultrasound and CT, respectively.
Abdominal fat may interfere with proper diagnosis in imaging modalities. In addition, in non-obese Chinese children, CT resolution was as good as ultrasound (17). Thus, abdominal $\mathrm{CT}$ is the best method for diagnosis of abdominal purpura in Chinese children but has the issue of radiation dose.

The use of an imaging method for the diagnosis of abdominal involvement in purpura presents many limitations and is only useful for some clinical presentations or complications. All children were subjected to laboratory tests with imaging modalities, which require exposure of high radiation doses. Indeed, the treatment period and treatment dose were small, yet too high for diagnosis methods. Demographical characteristics and seasons also have an impact over the diverse etiology of abdominal purpura in Chinese children $(18,19)$; 
however, the present study did not consider such parameters. Erosions, mural hematoma and ulcers can be visualized by endoscopy (10). Furthermore, endoscopic examinations can be more useful in view of the pathophysiology of the disease, but endoscopy was not performed at all. Additionally, serum immunoglobulin A was not used to detect abdominal purpura in the children (9). The justification for that the level of serum immunoglobulin $\mathrm{A}$ is varied with age and the other demographical and clinical characters. Other limitations of the present study include the retrospective nature of the analysis and lack of a randomized trial.

In conclusion, laboratory tests and clinical features do not rule out other etiologies of acute colicky pain. Ultrasound and CT had the same findings in the diagnosis of abdominal purpura; however, CT had an issue of radiation dose. Abdominal ultrasound is an easy, non-invasive and safe method for the detection of abdominal purpura.

\section{Acknowledgements}

Not applicable.

\section{Funding}

The present study was supported by a Provincial Health and Family Planning Commission-Initiated Fund Project (grant no. gzwjkj2015-1-067).

\section{Availability of data and materials}

The datasets used and/or analyzed during the current study are available from the corresponding author on reasonable request.

\section{Authors' contributions}

YG and SL contributed equally to the project administration and conceptualization, data analysis using software, formal analysis, validation, resources, data curation, investigation, and literature review of the study. YG and SL drafted, reviewed and edited the manuscript for intellectual content. Both authors read and approved the final version of the manuscript. Both authors agree to be accountable for all aspects of work ensuring integrity and accuracy.

\section{Ethics approval and consent to participate}

The protocol (approval no. GPH/CL/14/19; February 15, 2019) of the present study was approved by the Guiyang Maternal and Child Health Hospital review board. An informed consent form was signed by the parents of all participating children regarding diagnosis, radiology, treatment and anesthesia (if required) during hospitalization. The present study adheres to the law of China, the Strengthening the Reporting of Observational Studies in Epidemiology statement and the Declaration of Helsinki (2008).

\section{Patient consent for publication}

An informed consent form was signed by parents of all participating children regarding publication of the present study in all formats of the publication house, including personal data and images, irrespective of time and language.

\section{Competing interests}

The authors declare that they have no competing interests.

\section{References}

1. Başaran Ö, Cakar N, Uncu N, Celikel BA, Kara A, Cayci FS, Taktak A and Gür G: Plasma exchange therapy for severe gastrointestinal involvement of henoch schönlein purpura in children. Clin Exp Rheumatol 33 (Suppl 89): S176-S180, 2015.

2. Srivali N, Ungprasert P, Ahmed S, Cheungpasitporn W and Bischof EF: A case of childhood vasculitis presenting in adulthood. Am J Emerg Med 31: 254-255, 2013.

3. Lim CJ, Chen JH, Chen WL, Shen YS and Huang CC: Jejunojejunum intussusception as the single initial manifestation of Henoch-Schönlein purpura in a teenager. Am J Emerg Med 30: 2085, 2012.

4. McPartland $\mathrm{K}$ and Wright $\mathrm{G}$ : Acute abdominal pain: Henoch-Schönlein purpura case in a young adult, a rare but important diagnosis. Clin Med (Lond) 19: 77-79, 2019.

5. Zhang Q, Guo Q, Gui M, Ren Z, Hu B, Lu L and Deng F: Henoch-Schönlein purpura with acute pancreatitis: Analysis of 13 cases. BMC Pediatr 18: 159, 2018.

6. Yang YH, Yu HH and Chiang BL: The diagnosis and classification of Henoch-Schönlein purpura: An updated review. Autoimmun Rev 13: 355-358, 2014.

7. Keenswijk W, Van Renterghem K and Vande Walle J: A case report of a child with purpura, severe abdominal pain, and hematochezia. Gastroenterology 153: e10-e11, 2017.

8. Yang Z, Guo L, Xiong H, Gang Z, Li JX, Deng YP, Dawa QZ, PubuZXandLiH:Clinical analysis of childhoodHenoch-Schonlein purpura on the Tibetan Plateau, China. Zhongguo Dang Dai Er Ke Za Zhi 16: 1231-1235, 2014 (In Chinese).

9. Batu ED and Ozen S: Vasculitis: Do we know more to classify better? Pediatr Nephrol 30: 1425-1432, 2015.

10. Reamy BV, Williams PM and Lindsay TJ: Henoch-Schönlein purpura. Am Fam Physician 80: 697-704, 2009.

11. BMJ Best Practice. Evaluation of abdominal pain in children. https://bestpractice.bmj.com/topics/en-us/787/diagnosis-approach. Accessed December 1,2018.

12. Brody AS, Frush DP, Huda W and Brent RL; American academy of pediatrics section on radiology: Radiation risk to children from computed tomography. Pediatrics 120: 677-682, 2007.

13. Chang WL, Yang YH, Lin YT and Chiang BL: Gastrointestinal manifestations in Henoch-Schönlein purpura: A review of 261 patients. Acta Paediatr 93: 1427-1431, 2004.

14. Teng X, Gao C, Sun M and Wu J: Clinical significance of fecal calprotectin for the early diagnosis of abdominal type of Henoch-Schonlein purpura in children. Clin Rheumatol 37: 1667-1673, 2018.

15. Ozen S, Pistorio A, Iusan SM, Bakkaloglu A, Herlin T, Brik R, Buoncompagni A, Lazar C, Bilge I, Uziel Y, et al: Paediatric rheumatology international trials organisation (PRINTO). EULAR/PRINTO/PRES criteria for Henoch-Schönlein purpura, childhood polyarteritis nodosa, childhood wegener granulomatosis and childhood takayasu arteritis: Ankara 2008. Part II: Final classification criteria. Ann Rheum Dis 69: 798-806, 2010.

16. Kirpalani H and Nahmias C: Letter: Radiation risk to children from computed tomography. Pediatrics 121: 449-450, 2008.

17. Pelin M, Paquette B, Revel L, Landecy M, Bouveresse S and Delabrousse E: Acute appendicitis: Factors associated with inconclusive ultrasound study and the need for additional computed tomography. Diagn Interv Imaging 99: 809-814, 2018.

18. Chen O, Zhu XB, Ren P, Wang YB, Sun RP and Wei DE: Henoch schonlein purpura in children: Clinical analysis of 120 cases. Afr Health Sci 13: 94-99, 2013.

19. Liu LJ, Yu J and Li YN: Clinical characteristics of Henoch-Schönlein purpura in children. Zhongguo Dang Dai Er Ke Za Zhi 17: 1079-1083, 2015 (In Chinese). 